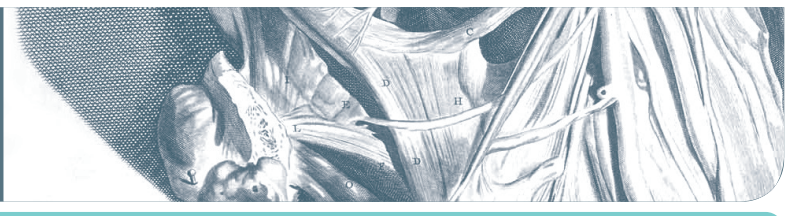

\title{
Síndrome de Klippel - Trenaunay - Weber. Reporte de caso
}

\section{Klippel - Trenaunay - Weber syndrome. Case report}

Julián Camilo Velásquez-Paz ${ }^{1,2}$; Angélica Rocío Álvarez-Mina ${ }^{1}$; Andrés Felipe Andrade-Eraso ${ }^{1,2}$, Jenny Adriana Morán-Fernández; Johana Marcela Morán-Fernández¹.

\section{RESUMEN}

Objetivo: Caracterizar clínicamente un caso de Síndrome de Klippel-Trenaunay - Weber (SKTW). Descripción del caso: Presentamos a una paciente de 22 ańos de edad con antecedente de epilepsia con manejo irregular, que consultó porcrisis convulsiva además de cuadro respiratorio infeccioso. Al abordarla hay evidencia de malformaciones vasculares, manchas cutáneas en vino de oporto e hipertrofia de tejidos blandos en hemicuerpo izquierdo, se realizan ecografias de tejidos blandos de miembros inferiores, tóraxy abdomen que

\begin{abstract}
Objective: To characterize a case of Klippel-Trenaunay-Weber Syndrome (KTW). Case description: We present a 22-year-old patient, with a history of epilepsy with irregular treatment, who consulted for a seizure in addition to an infectious respiratory condition, when approaching her, there is evidence of vascularmalformations, portwine skin stains and soft tissue hypertrophy in the left hemibody, a soft tissue ultrasound of the lower limbs, thorax and abdomen are performed, that reported multiple venous malformations and hypertrophy of
\end{abstract}

\section{Historial del artículo:}

Fecha de recepción: $\quad$ 20/01/2020

Fecha de aceptación: 10/04/2020

1 Hospital Universitario San José, Popayán, Colombia.

2 Departamento de Medicina Interna, Facultad de Ciencias de la Salud, Universidad del Cauca, Popayán, Colombia.

Correspondencia: Julián Camilo Velásquez-Paz. Departamento de Medicina Interna. Hospital Universitario San José. Cra. 6 No. 10N142, Popayán, Colombia. E-mail: jcvp25@hotmail.com

Como citar este artículo: Velásquez-Paz J, Álvarez-Mina A, Andrade-Eraso A, Morán-Fernández JA, Morán-Fernández JM. Síndrome de Klippel - Trenaunay - Weber. Reporte de caso. Revista de la Facultad de Ciencias de la Salud de la Universidad del Cauca. 2020; 22 (2):60-64. https:// doi.org/10.47373/rfcs.2020.v22.1423 
reportaron múltiples malformaciones venosas e hipertrofia de mama y extremidades izquierdas. Con estos hallazgos asociados a las características clínicas, se hizo diagnóstico de SKTW. Durante su estancia hospitalaria se realizó manejo anticomicial y antibiótico; se solicitaron estudios imagenológicos complementarios que no fueron llevados a cabo por lapaciente. Conclusiones: Es importante dar visibilidad a estapatologíapor la importante morbimortalidad quegenera. Debe realizarse un diagnóstico y tratamiento oportuno, a cargo de un equipo multidisciplinario especializado debido a la posible afectación de múltiples órganos, con el fin de disminuir las complicaciones y mejorar la calidad de vida de quieneslapadecen.

Palabras claves: Sindrome de Klippel -Trenaunay -Weber; malformaciones vasculares; mancha Vino de oporto; epilepsia; hipertrofia (DeCS).

\section{INTRODUCCIÓN}

El síndrome de Klippel-Trenaunay - Weber (SKTW), descrito en la primera década del siglo XX por Maurice Klippel, Paul Trenaunay y Frederick Parkes Weber, es una anomalía congénita poco frecuente, de etiología desconocida, probablemente asociado con la mutación del gen VG5Q y relacionado con el crecimiento vascular (1). Se caracteriza por la triada de anomalías vasculares, manchas cutáneas en vino de oporto e hipertrofia de tejidos blandos y óseos, aunque al cuadro clínico pueden agregarse fístulas arteriovenosas y hemangiomatosis visceral. El compromiso es unilateral en la mayoría de los casos $(2,3)$.

\section{HERENCIA Y ASPECTOS MOLECULARES}

No se conoce un patrón de herencia definido para el síndrome, se considera que tiene una expresión genética variable y que puede ser transmitida durante muchas generaciones pasando desapercibida (3,4). Algunos estudios genéticos han identificado tres anormalidades cromosómicas en pacientes con SKTW: dos traslocaciones balanceadas $\mathrm{t}(5.11)(\mathrm{q} 13.3$; p15.1) y $\mathrm{t}(8,14)(\mathrm{q} 22.3$; q13) y un anillo extra supernumerario en el cromosoma 18 (5). Se ha asociado a la mutación del gen VG5Q, formalmente llamado AGGF1 (cuyo locus es 5q13.3), y a polimorfismos de un solo nucleótido en AGGF1, rs7704267 y rs13155212, con riesgo de SKTW al expresarse ampliamente en varios tipos de células endoteliales, osteoblastos y en células musculares lisas, resultando en un crecimiento excesivo asimétrico de estos componentes (5). Otros genes que pueden verse afectados son el RASA1, AKT1 y PIK3CA (6). the breast and left extremities, with these findings associated with the clinical characteristics, a diagnosis of SKTW is made, during their hospital stay anticonvulsant and antibiotic management is administered, complementary imaging studies are requested that are not completed by the patient. Conclusions: It is important to give visibility to this pathology due to the significant morbidity and mortality it generates in order to carry out a timely diagnosis and treatment, in order to reduce complications and improve the quality of life of those who suffer from it; in addition to being accompanied by a specialized multidisciplinary team due to the possible involvement of multiple organs.

Keywords: Klippel-Trenaunay-Weber syndrome; vascular malformations; Port-Wine Stain; epilepsy; hypertrophy (MeSH).

\section{EPIDEMIOLOGÍA}

La información epidemiológica del síndrome es escasa; sin embargo, se conoce que ocurre aproximadamente en 1 de cada 20.000 a 40.000 nacidos vivos, no tiene predilección por el sexo ni por etnia, se presenta principalmente en la infancia, afecta en la mayoría de los casos las extremidades inferiores seguidas por las extremidades superiores, tronco, cabeza y cuello, generalmente afecta el lado derecho del cuerpo y un solo cuadrante corporal. La malformación más frecuentemente encontrada en estos pacientes son las vasculares y capilares en el 80 - 98\% de los casos, luego las várices en el 70 - 80\% de los casos. El hallazgo más variable es la hipertrofia de las extremidades en el 50 - 94\% (7).

\section{CASO CLÍNICO}

Mujer de 22 ańos de edad, mestiza, sin ocupación, con antecedente de epilepsia en manejo farmacológico irregular con risperidona y valproato de magnesio, además de hospitalizaciones frecuentes por epilepsia descompensada e infecciones respiratorias bajas a repetición. La paciente es producto de la tercera gestación de una madre G3P2C1V3, nacida por cesárea por parto estacionario, de una unión no consanguínea (Figura 1). Su nacimiento fue a término, sin complicaciones periparto; la madre no refiere infecciones en el embarazo, exposición a drogas, agentes químicos o físicos. No hay información sobre movimientos fetales durante el embarazo, parto, presentación del feto, APGAR, datos antropométricos al nacer, cordón umbilical, placenta o desarrollo psicomotor. Paciente sin antecedentes de familiares con diagnóstico de SKTW (Figura 1). 
Figura 1. Genealogía.

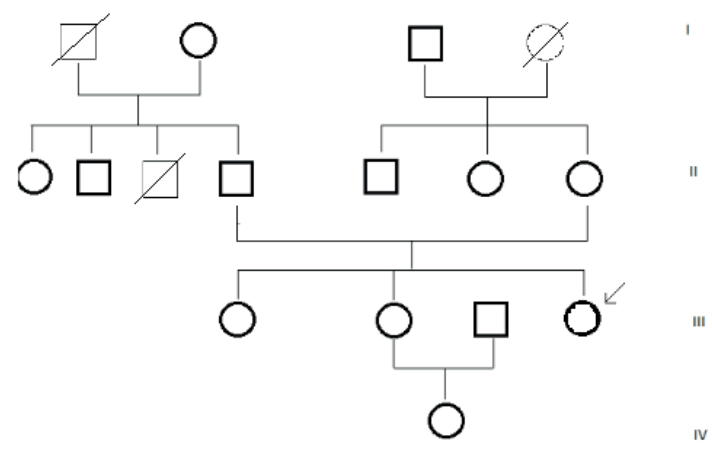

La paciente consultó por convulsión tónica clónica generalizada con hemiparesia de hemicuerpo izquierdo en el postictal. Fue valorada en primera instancia por medicina interna, especialidad encargada de su seguimiento hasta el egreso, quién administró anticomicial endovenoso con fenitoína y posteriormente manejo oral con valproato de magnesio (medicamento conciliado), tratamiento con el cual no presentó nuevos eventos convulsivos durante la hospitalización. Al ingreso, además, presentó evidencia paraclínica de neumonía adquirida en la comunidad, sin necesidad de manejo avanzado de la vía aérea, que requirió de esquema antibiótico durante siete días con cefepime. Por la convulsión presentada, hubo evidencia de rabdomiólisis e insuficiencia renal aguda secundaria que fue abordada y resuelta con manejo hidroelectrolítico con lactato de Ringer. Tuvo una franca evolución hacia la mejoría durante su estancia hospitalaria.

El fenotipo evidenciaba hipertrofia de hemicuerpo izquierdo, con manchas cutáneas en vino de oporto en cabeza, región superior del tórax y miembro inferior izquierdo(Figuras 2,3 y4) $\mathrm{y}$ várices superficiales no tronculares en extremidad inferior ipsilateral; presentaba alteración en medida de la brazada, distancia intercántica interna, filtrum y perímetro cefálico. Según familiar, la paciente presentaba estas alteraciones desde los dos ańos de edad aproximadamente. Se realizaron paraclínicos: Cuadro hemático, electrolitos y tiempos de coagulación dentro de límites normales, tomografía axial computarizada simple de cerebro sin alteraciones, azoados elevados en resolución respecto al ingreso, ecografía de tejidos blandos de miembros inferiores, tórax y abdomen reportaron múltiples malformaciones venosas e hipertrofia. Paciente valorada por genética clínica, quien realizó diagnóstico clínico de SKTW por presencia de dos criterios mayores y dos menores. Se consideró diagnóstico diferencial con síndrome de Proteus. Cabe resaltar que el diagnóstico retrasado de este síndrome en la paciente ocurre por domicilio lejano y renuencia a la consulta especializada.
Figura 2. Imagen frontal, presencia de manchas en vino de oporto e hipertrofia de hemicara izquierda.

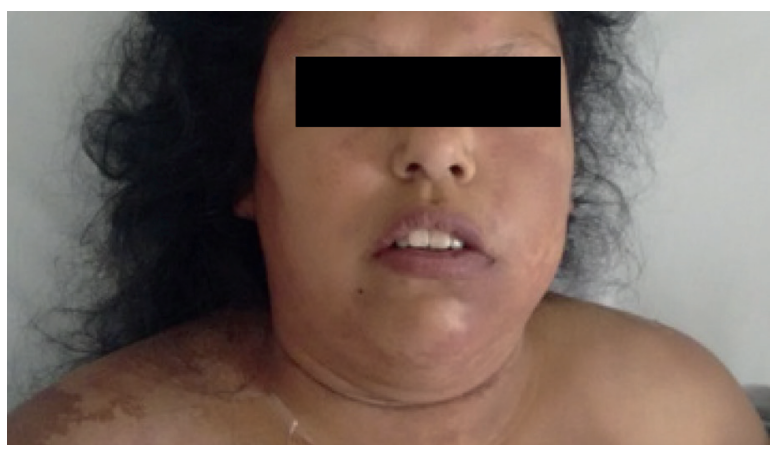

Figura 3. Tórax anterior, presencia de nevus flammeus en región superior e hipertrofia de mama izquierda.

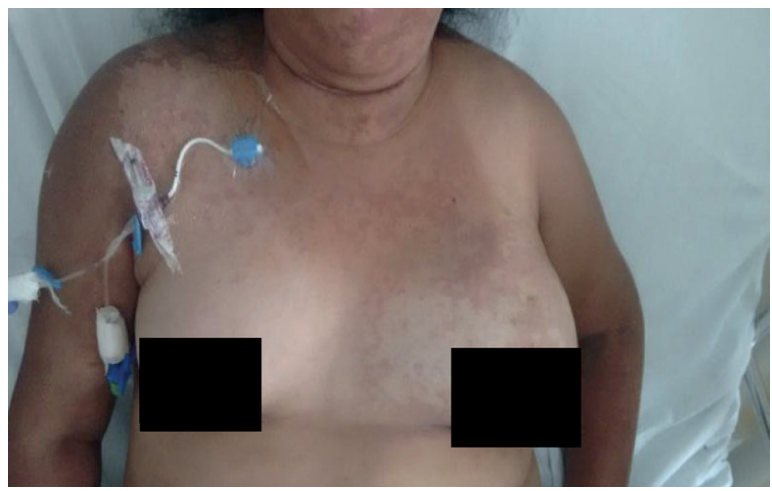

Figura 4. Hemiabdomen y miembro inferior izquierdo con hipertrofia, además manchas en vino de oporto y dilatación varicosa superficial en miembro inferior ipsilateral.

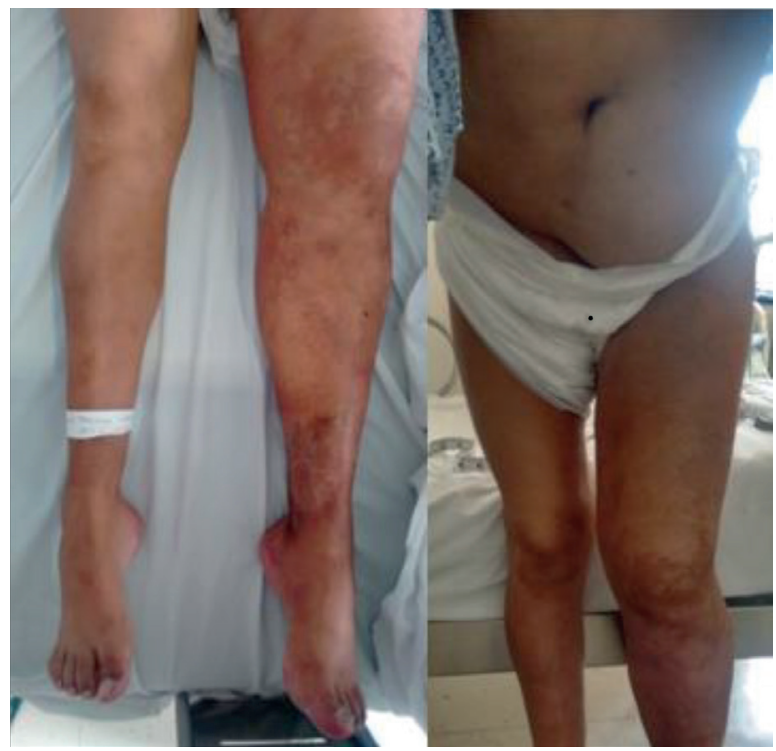


Posterior al diagnóstico, se dio orden de continuar estudios imagenológicos cerebrales (Resonancia magnética nuclear y angiografía cerebral), continuar manejo anticonvulsivante, recomendaciones generales y signos de alarma para consultar al servicio de urgencias; sin embargo, la paciente no asistió a los demás estudios especializados solicitados ni a controles médicos.

\section{DISCUSIÓN}

El síndrome de Klippel-Trenaunay - Weber se caracteriza por una triada clínica de malformaciones vasculares, hipertrofia de tejidos blandos y manchas cutáneas en vino de oporto (7). Su diagnóstico puede hacerse de manera clínica, cuando las alteraciones son evidentes (8). Se han descrito criterios mayores: malformaciones capilares, que incluye la mancha de color vino de oporto, malformaciones venosas (que pueden ser: venas muy pequeńas o ausentes, persistencia de venas fetales, varices, aumento de tamańo de las venas, venas tortuosas y malformaciones valvulares de las venas), malformaciones arteriovenosas (que incluyen solamente las malformaciones arteriovenosas muy pequeńas o fístulas arteriovenosas) y malformaciones linfáticas; y criterios menores: Crecimiento anormal en la longitud o grosor del hueso y crecimiento anormal del tejido blando en la longitud o grosor (9); requiriéndose dos criterios mayores y por lo menos uno menor para el diagnóstico del SKTW. En el caso presentado se evidencia la presencia de dos criterios mayores (malformaciones capilares tipo mancha vino de oporto y varices en miembro inferior izquierdo) y los dos menores (se manifiesta con hipertrofia de hemicuerpo izquierdo). El diagnóstico fue clínico, debido a las malformaciones evidentes de la paciente, y confirmado por expertos en el área de genética clínica.

En un estudio realizado en 2013, Sreekar et. al, describieron 19 casos de pacientes con SKTW desde 2001-2010, con una edad promedio de presentación a los 19 ańos, siendo todas mujeres, en quienes la mayoría de manifestaciones obedecieron a edema de miembros inferiores y presencia de varices venosas (10). Respecto al presente caso se encuentran varias similitudes, en referencia al grupo etario y sexo de la paciente, además con la presentación clínica ya mencionada.

Los diagnósticos diferenciales incluyen aquellas malformaciones que generan sobrecrecimiento y malformaciones vasculares como los síndromes de Proteus (que se consideró y se descartó en la paciente por genética clínica), de Sturge-Weber, de Parkes-Weber, de Maffuci, de sobrecrecimiento lipomatoso asociado a malformaciones vasculares (CLOVES), de tumor hamartoma y la anomalía fibroadiposovascular (FAVA) (11).

El diagnóstico y el compromiso se deben profundizar con estudios imagenológicos, por ejemplo, la ecografía doppler para evaluar fístulas arteriovenosas, la flebografía de contraste y la resonancia magnética que muestran el grado de hipertrofia tisular, el compromiso óseo y confirman las anormalidades relacionadas con el sistema linfático (11). Dado que la presentación del síndrome es variable, es importante la investigación de hemangiomas viscerales a nivel cerebral, ocular o del tracto intestinal y genitourinario. Para la paciente no fue posible descartar compromisos sistémicos debido al abandono del seguimiento médico; se esperaba determinar si existían anormalidades vasculares a nivel del sistema nervioso central que podían condicionar crisis convulsivas, al tratarse de una paciente que ingresa frecuentemente por episodios ictales.

La severidad del SKTW puede determinarse según el tipo de displasia vascular venosa, arterial o mixta. Esto condiciona algunas manifestaciones clínicas como cuadros de hemorragias de vías digestivas, siendo los sangrados variceales esofágicos, colónicos distales o de recto los más frecuentes, también se describe compromiso genitourinario (12-14). Lo anterior no se documenta en la paciente y, por ende, no se considera un caso severo.

El tratamiento del SKTW debe ser multidisciplinario y dirigido a evitar las complicaciones agudas y crónicas, así como a prevenir secuelas. En el presente caso, la corrección de las alteraciones agudas, por medio de manejo anticomicial y de las complicaciones infecciosas y renales, fueron la piedra angular del restablecimiento de las condiciones basales de la paciente. Cuando los afectados se tornan excesivamente sintomáticos, el manejo se enfoca en la realización de procedimientos vasculares tales como embolización, escleroterapia, resección de las displasias y corrección de fístulas arteriovenosas (15); sin embargo, aquellos pacientes asintomáticos y sin complicaciones secundarias a las malformaciones vasculares, pueden ser manejados de manera conservadora (11).

El SKTW es una entidad poco frecuente, lo que condiciona que se tenga poca información y que, por su diversa sintomatología, podría pasar desapercibido entre el personal de salud. Es importante que los profesionales realicen un examen físico detallado de pacientes que cursan con lesiones cutáneas de características angiomatosas, discretas asimetrías faciales o corporales, además de hacer énfasis en síntomas asociados que podrían convertirse en un signo de alarma 
con el fin de su identificación temprana, para asegurar un manejo integral y así mismo prevenir y tratar complicaciones secundarias. También se recomienda sensibilizar a los pacientes acerca de la enfermedad y de la importancia de la consulta y seguimiento.

Es importante dar visibilidad a esta patología por la importante morbimortalidad que puede representar para los afectados; si bien no existe tratamiento curativo, existen objetivos terapéuticos destinados a mejorar los síntomas del paciente y prevenir consecuencias graves de la enfermedad. Quienes padecen este síndrome deben acompańarse de un equipo multidisciplinario especializado debido a la posible afectación de múltiples órganos.

\section{DECLARACIÓN DE CONFLICTOS DE INTERESES}

Los autores declaran no tener conflictos de intereses.

\section{ACLARACIÓN DE CONSENTIMIENTO INFORMADO}

Se obtuvo el aval del paciente y su familiar para la publicación de este caso.

\section{REFERENCIAS}

1. Patel AL, Shaikh WA, Saini KS, Madhusudan YA. Klippel Trénaunay syndrome. J Assoc Physicians India. 2006;54:156-8.

2. Martínez ME, Avendańo GMLG, García RE, Cruz JG de la. Síndrome de Klippel-Trenaunay. Hallazgos clínicos y de imagen. Anales de Radiología México. 2006;5(3):245-51.

3. Boló H, Ortiz-C LM, Samudio-D GC, Ruiz-Diaz J. Síndrome de Klippel-Trenaunay asociado a litiasis renal. Reporte de un caso pediátrico y revisión de la literatura. Pediatr (Asunción). 2013;40(3):247-51.

4. Velasco PFP, Guerra G, Yusef A. Rectorragía en el Síndrome de Klippel-Trenaunay-Weber: Presentación de un caso. CIMEL Ciencia e Investigación Médica Estudiantil Latinoamericana. 2008;13(2):73-7.

5. Hu Y, Li L, Seidelmann SB, Timur AA, Shen PH, Driscoll DJ, et al. Identification of association of common AGGF1 variants with susceptibility for Klippel-Trenaunay syndrome using the structure association program. Ann Hum Genet. Septiembre de 2008;72(Pt 5):636-43.
6. Tian XL, Kadaba R, You SA, Liu M, Timur AA, Yang L, Chen Q, Szafranski P, Rao S, Wu L, Housman DE, DiCorleto PE, Driscoll DJ, Borrow J, Wang Q. Identification of an angiogenic factor that when mutated causes susceptibility to Klippel-Trenaunay syndrome. Nature. 2004; 427(6975):640-5.

7. Husmann DA, Rathburn SR, Driscoll DJ. Klippel-Trenaunay syndrome: incidence and treatment of genitourinary sequelae. J Urol. abril de 2007;177(4):1244-9.

8. Avilés Izquierdo JA, Suárez Fernández R, Lázaro Ochaíta P, Recarte García-Andrade C. Síndrome de KlippelTrenŕunay en el adulto. An. Med. Interna (Madrid). 2003;20(11):56-7.

9. Janniger CK. Klippel-Trenaunay-Weber Syndrome. Medscape Reference. 2016; http://emedicine.medscape. com/article/1084257-overview.

10. Sreekar H, Dawre S, Petkar KS, Shetty RB, Lamba S, Naik $S$, et al. Diverse manifestations and management options in Klippel-Trenaunay syndrome: a single centre 10-year experience. J Plast Surg Hand Surg. septiembre de 2013;47(4):303-7.

11. Gontero R, Ortiz A, Roverano S, Paira S. Síndrome KlippelTrenaunay: comunicación de dos casos. Rev argent reumatol. 2017;42-7.

12. Samo S, Sherid M, Husein H, Sulaiman S, Yungbluth M, Vainder JA. Klippel-Trenaunay Syndrome Causing LifeThreatening GI Bleeding: A Case Report and Review of the Literature. Case Rep Gastrointest Med 2013;2013:813653.

13. Kocaman O, Alponat A, Aygün C, Gürbüz Y, Sarisoy HT, Celebi A, et al. Lower gastrointestinal bleeding, hematuria and splenic hemangiomas in Klippel-Trenaunay syndrome: a case report and literature review. Turk J Gastroenterol. 2009;20(1):62-6.

14. Sharma D, Lamba S, Pandita A, Shastri S. KlippelTrénaunay Syndrome - A Very Rare and Interesting Syndrome. Clin Med Insights Circ Respir Pulm Med. 2015;9:1-4.

15. Lee BB, Do YS, Byun HS, Choo IW, Kim DI, Huh SH. Advanced management of venous malformation with ethanol sclerotherapy: Mid-term results. Journal of Vascular Surgery. 2003;37(3):533-8. 